\title{
Réformes et changements en éducation en Slovénie
}

\section{Slavko Gaber et Veronika Tašner}

Traducteur : Valérie Téhio

\section{(2) OpenEdition \\ Journals}

Édition électronique

URL : http://journals.openedition.org/ries/1010

DOI : 10.4000/ries. 1010

ISSN : 2261-4265

Éditeur

Centre international d'études pédagogiques

Édition imprimée

Date de publication : 1 septembre 2010

Pagination : 171-176

ISBN : 978-2-85420-582-4

ISSN : $1254-4590$

\section{Référence électronique}

Slavko Gaber et Veronika Tašner, «Réformes et changements en éducation en Slovénie », Revue internationale d'éducation de Sèvres [En ligne], 54 I septembre 2010, mis en ligne le 01 septembre 2013, consulté le 20 avril 2019. URL : http://journals.openedition.org/ries/1010 ; DOI : 10.4000/ries.1010

Ce document a été généré automatiquement le 20 avril 2019

(C) Tous droits réservés 


\title{
Réformes et changements en éducation en Slovénie
}

\author{
Slavko Gaber et Veronika Tašner
}

Traduction : Valérie Téhio

1 En avril 2009, la Slovénie ${ }^{1}$ a réuni un groupe d'experts nationaux chargé de préparer un rapport qui servirait de « base à des changements systémiques et, sur la base de preuves, proposerait une amélioration du système éducatif » (Lukšǐc 2009). Le ministre attend ces analyses et propositions de changement pour 2011.

2 Bien que la Slovénie ne soit que récemment devenue un pays indépendant (1991), ce n'est pas la première fois qu'elle s'engage de manière systématique dans une politique de changement en éducation. On peut considérer que la réforme éducative a accompagné le processus d'indépendance. Les réformes conduites dans les années quatre-vingt-dix et les réformes antérieures des années quatre-vingts forment, pour une grande part, la structure et la dynamique d'un système éducatif relativement stable et efficient ${ }^{2}$ ainsi que d'un réexamen récurrent des besoins de changement pour s'adapter au présent.

3 L'origine de la structure du système éducatif actuel, de ses forces et faiblesses et des besoins de changement remonte à l'époque de l'ancien régime. Les dix dernières années de socialisme avaient conduit à des restructurations de la société et de son organisation politique ainsi qu'à un réexamen de la logique et de la validité de l'éducation socialiste. Les enseignants et les chefs d'établissements avaient lancé de nombreux projets innovants. De plus, l'un des plus importants «projets » préconisé par l'opposition au précédent gouvernement était de rétablir les gymnasia (lycées d'enseignement général), qui avaient été formellement abolis, de réintroduire la matura (baccalauréat) et de substituer l'enseignement de la sociologie et de la culture civique aux anciens cours d'éducation civique (conduite de soi et marxisme). La chambre des petites et moyennes entreprises demandait, pour sa part, la réintroduction d'un système par alternance, l'université avait entrepris de préparer une loi sur l'enseignement supérieur, etc.

4 Le pays nouvellement établi commença par réformer l'éducation en tant qu'élément structurant les attentes nationales, à savoir l'indépendance, la transition vers une 
démocratie parlementaire représentative et vers un système pluriel de valeurs, les efforts pour intégrer l'Europe combinés au développement de la sphère productive et aux changements requis dans les disciplines scientifiques.

5 Les réformes éducatives des années quatre-vingt-dix se sont ainsi inscrites dans un environnement soucieux de changement. Cependant, comme partout en Europe, les changements qui se sont produits et étaient censés être appuyés par les citoyens, les parents et les enseignants, n'ont pas manqué de rencontrer de l'opposition, "chez ces mêmes politiciens, enseignants ou parents $»^{3}$.

\section{Quelques caractéristiques du système éducatif actuel}

Le "nouveau » système éducatif, né d'une réforme planifiée avec précision, a été construit autour d'une forte composante préscolaire. L'éducation pré-primaire n'est pas obligatoire en Slovénie. Elle est assurée par des institutions préscolaires publiques ou privées. Les Kindergarten sont publics, mais leur organisation et leur financement sont de la responsabilité des municipalités. Les établissements pré-primaires offrent des soins à la petite enfance dans une perspective éducative. Les enfants sont accueillis dans des Kindergarten à partir de 11 mois et peuvent y rester jusqu'à l'âge de la scolarité obligatoire (6 ans). Les enfants sont divisés en deux grandes classes d'âge : de 11 mois à 3 ans et audelà de 3 ans. Le premier groupe peut accueillir entre 10 et 12 enfants, selon la diversité des âges des enfants accueillis. Dans le second groupe, 17 à 22 enfants peuvent être accueillis par deux enseignants pendant environ six heures par jour.

7 À 6 ans, les enfants commencent la scolarité obligatoire intégrée. C'est une nouveauté : auparavant, les enfants commençaient à 7 ans. La durée de la scolarité obligatoire est passée de huit à neuf années. L'éducation est gratuite pour tous. Les parents peuvent choisir entre des écoles publiques et privées ou encore éduquer leurs enfants à la maison, s'ils apportent la preuve que l'enfant atteint les standards de l'éducation de base.

La scolarité obligatoire est divisée en trois cycles. Au cours du dernier cycle, les enseignements de langue étrangère, de langue et de mathématiques font l'objet d'une différenciation de l'enseignement et de l'évaluation en trois niveaux. Les établissements scolaires sont également tenus d'offrir des options obligatoires en sciences humaines et sociales, en sciences naturelles et en technologie, avec un minimum de trois options par champ disciplinaire. À partir de 2008, une seconde langue étrangère obligatoire a été progressivement implantée (au troisième cycle); à compter de 2012, tous les élèves de l'enseignement de base devront apprendre deux langues étrangères.

Après l'éducation de base intégrée, plusieurs voies sont offertes aux élèves :

- deux ou trois années d'enseignement professionnel diversifié, en coopération avec le secteur industriel; il faut noter que l'enseignement professionnel accueille des cohortes et une proportion d'élèves en diminution ( $16 \%$ d'une classe d'âge) ;

- quatre années d'enseignement technologique, à la fois diversifié et généraliste : cette filière accueille plus de $40 \%$ d'une classe d'âge ;

- avec la réinstauration de la matura et du gymnasium, le programme le plus généraliste de l'enseignement secondaire supérieur, d'une durée de quatre ans, est celui qui connaît la plus rapide croissance. Actuellement, il accueille plus de $40 \%$ des élèves, avec un ratio de quarante garçons pour soixante filles. Le programme est diversifié dans ses orientations, 
allant, de façon classique, du plus artistique au plus scientifique et incluant au moins deux langues étrangères.

10 Les élèves qui réussissent le baccalauréat national externe (matura) poursuivent leur éducation dans une université slovène. L'Université de Ljubljana - la plus importante avec 56000 étudiants - est aussi la mieux classée ${ }^{4}$.

11 En bref, les réformes ont abouti à un système éducatif relativement moderne et stable.

\section{Les enjeux du développement du système éducatif}

12 Aujourd'hui, la Slovénie dispose d'un système structuré, différencié et efficient. Cependant, il semble qu'elle ait besoin :

13 Pour analyser l'état actuel de l'éducation et les enjeux de son développement futur, nous utiliserons les critères suivants: l'accès (le niveau de libéralisation/démocratisation), l'équité, le financement, l'efficience, le rapport entre éducation privée et publique.

\section{L'accès}

14 En Slovénie, $82 \%$ des enfants de plus de 3 ans et $90 \%$ de ceux de 5 ans sont accueillis dans le pré-primaire. Cependant, le taux d'intégration des moins de 3 ans est de $45 \%$ et de $66,3 \%$ pour les enfants de 3 ans, alors que la moyenne européenne est de 72,3\% pour les enfants de 3 ans (ECE 2009, 68). Toute la classe d'âge concernée intègre l'éducation de base (entre 6 et 15 ans) et $98 \%$ de ceux qui atteignent la dernière année de l'éducation de base continuent dans le secondaire supérieur. Le niveau d'intégration dans le tertiaire est élevé, avec plus de $65 \%$. Cependant l'objectif national pour la prochaine décennie est de plus de $70 \%$. Un autre point important est le nombre croissant d'étudiants inscrits en troisième cycle ( 3006 en 1998/1999; 8344 en 2005/2006). Il en est de même pour l'éducation des adultes : 14,8\% de la population entre 25 et 64 ans est concernée par l'éducation tout au long de la vie, quand la moyenne européenne est de 9,5\%. L'accès et l'intégration dans le système scolaire slovène sont donc moins liés au statut social des familles que dans la majorité des pays européens.

L'accès à l'éducation est équitable grâce à un réseau diversifié et étendu de Kindergarten et d'établissements scolaires. Les infrastructures d'éducation supérieure et des adultes sont également disponibles. La mise en place d'une offre éducative dans les zones isolées est coûteuse, mais il est nécessaire de maintenir le niveau d'offre existant, afin de garantir une implantation décentralisée dans le pays. La Slovénie peut assumer ce niveau d'offre éducative.

En lien avec une politique d'accès ouvert, les décideurs politiques dans le domaine de l'éducation sont aujourd'hui confrontés à :

\section{Équité}

En termes de qualité, la Slovénie est relativement moins performante (PISA 2006). Les résultats scolaires dépendent encore trop de l'origine sociale de l'élève. Les données disponibles montrent que des taux d'intégration élevés sont nécessaires mais insuffisants pour atteindre un haut niveau d'égalité et d'équité. Un appui complémentaire (en termes d'opportunités) donné aux élèves qui ont un faible capital culturel, le plus précocement 
possible, est nécessaire pour diminuer l'impact de l'origine sur les résultats obtenus. Le système éducatif devrait être capable d'aider les élèves venant d'environnements linguistiques moins favorables pour les aider à améliorer leurs compétences linguistiques ; il devrait fournir un appui financier aux élèves migrants, des repas chauds, un accès gratuit et subventionné aux manuels, aux concerts, au théâtre, aux dortoirs, etc., autant de mesures qui devraient être renforcées et mieux ciblées.

\section{Financement}

Depuis plus de dix ans, la Slovénie alloue 5,8 à $6 \%$ de son PIB à l'éducation. Cela la positionne parmi les systèmes éducatifs relativement bien dotés (la moyenne européenne étant de 5,03\%, selon Eurostat) $)^{5}$. Les ressources publiques sont également plutôt correctement réparties entre les niveaux du préscolaire $(0,52 \%)$, du primaire $(2,60 \%)$, du secondaire supérieur ( $1,45 \%)$ et du supérieur $(1,26 \%)$.

Il semble qu'il serait préférable d'étendre le dispositif budgétaire de financement forfaitaire de l'enseignement supérieur et secondaire supérieur à l'enseignement primaire et pré-primaire. Si cette décision était prise, l'objectif de cette extension du financement forfaitaire serait de promouvoir une plus grande autonomie de l'école et du Kindergarten et non de participer à la politique néolibérale de réduction des budgets alloués à l'éducation. Afin de consolider un système éducatif stable, efficient et juste en Slovénie, le budget de l'éducation devrait être augmenté de 0,2 à $0,3 \%$. Ces ressources nouvelles serviraient à réduire les coûts de la scolarité pré-primaire pour les parents de la classe moyenne (ceux des classes sociales défavorisées bénéficiant déjà d'aides), à diminuer les frais des étudiants qui travaillent à temps partiel pour financer leurs études, ainsi qu'à des mesures visant à une plus grande équité, comme mentionné plus haut. Il faut savoir que les familles slovènes supportent encore plus de $25 \%$ des coûts dans le préprimaire et qu'environ $20 \%$ des étudiants travaillent à temps partiel pour financer leurs études.

\section{Efficience}

Les données concernant l'efficience de l'éducation, en termes de mécanismes de socialisation de la jeunesse dans la société, démontrent la réussite du système. La violence scolaire, les délits juvéniles, la consommation de drogue, la solidarité, le souci de l'environnement et d'autres indicateurs permettent de revendiquer un relatif succès dans l'articulation entre l'enseignement pré-scolaire et scolaire et l'éducation familiale.

21 Les données relatives au niveau de connaissances sont moins convaincantes, même si les résultats sont loin d'être négatifs. La Slovénie doit viser de meilleurs résultats, si elle veut atteindre son objectif de rejoindre, à plus ou moins moyen terme, le tiers supérieur des pays membres de l'Union européenne, en termes de qualité de vie. La Slovénie atteint les résultats visés en science mais ses résultats restent seulement moyens en mathématiques et en langue maternelle. La situation est encore moins prometteuse si l'on considère que les étudiants ont tendance à surestimer le niveau de leurs connaissances. Pour atteindre de meilleurs résultats, il faut d'une part investir davantage dans l'éducation des populations socialement défavorisées et d'autre part élever le niveau des attentes des enseignants, des élèves, des parents et des responsables politiques. 

relativement bonne qualité. S'y ajoutent les écoles pré-scolaires et scolaires privées et cofinancées par le public. Chaque établissement présentant un programme adéquat et un nombre suffisant d'inscrits reçoit (automatiquement) $85 \%$ du financement par élève des écoles publiques. Si un Kindergarten ou une école est nécessaire pour compléter un réseau d'établissements publics à un niveau national ou local, il obtient $100 \%$ de son financement. Les initiatives privées en éducation évoluent progressivement. Elles sont plus importantes dans le champ du préscolaire et du supérieur et connaissent une bonne réussite dans l'enseignement secondaire. Il est vraisemblable qu'elles se développeront au niveau de l'éducation de base également. Le principal financeur de l'enseignement privé, exception faite de l'enseignement supérieur, est l'Église catholique. Nous pensons que la Slovénie n'a pas besoin de financer sur fonds publics $100 \%$ du coût des écoles privées. Le gouvernement devrait d'abord garantir la qualité des établissements publics. Cette attention devrait particulièrement se porter sur la diminution des effectifs d'élèves par classe.

Dans le supérieur, l'État devrait d'abord garantir le financement des universités publiques. Il devrait aussi procéder au financement des établissements universitaires privés par des mécanismes de concessions mais actuellement, il n'est pas nécessaire de prévoir un financement public supplémentaire pour les universités privées ni de faire financer les facultés privées par les facultés publiques, comme l'envisageait le gouvernement précédent. 
Quelques données en 2009

\begin{tabular}{|l|l|}
\hline Superficie : Nombre d'habitants : & $\begin{array}{l}20,273 \mathrm{~km}^{2} \\
2003358 \text { habitants }\end{array}$ \\
\hline Revenu moyen en mars 2009: & $1425,05 €($ brut $) ; 921,69 €($ net $)$ \\
\hline PIB : croissance au 4 trimestre : & $-0,8 \% ;$ per capita $18196 €(2008)$ \\
\hline
\end{tabular}

$1,229 \mathrm{~km}$ de lignes de chemin de fer ; 20,172 km de routes; 140237 entreprises ; 378 institutions de recherche ; 4779 clubs de sport ; 114794 étudiants ; 61 bibliothèques publiques ; 23 théâtres professionnels ou amateurs ; 55 musées ; 840000 usagers réguliers d'Internet (juin 2006) ; 996 185 souscripteurs de téléphones mobiles (juin 2009).

Source : Bureau des statistiques de la République de Slovénie : www.stat.si/.

\section{BIBLIOGRAPHIE}

EURYDICE (2009) : Early Childhood Education and Care in Europe: Tackling Social and Cultural Inequalities (ECE 2009), Bruxelles: Education, Audiovisual and Culture Executive Agency.

EURYDICE (2005) : Les chiffres clés de l'éducation en Europe, Luxembourg: OOPEC.

GABER S. (2005) : « Education, Mobility and Reproduction of Social Inequality », Analysis of Educational Policies in a Comparative Educational Perspective, Linz: Trauner Verlag.

GABRŠC``EK S. (ed.), DIMC N. (ed.) : Strategies of educational reform in south east Europe countries: proceedings of the seminar, Bled, Slovenia, June 8-10 2000. Ljubljana: Open Society Institute Slovenia: CPZ International Centre for Knowledge Promotion, 2000, pp. 55-68. http://www.seeeducoop.net/portal/id_slo.htm .

LUKSIC I. (2009) : Décision du ministère de l'Éducation, Ljubljana : MŠš.

OCDE (2007) : Regards sur l'éducation : les indicateurs de l'OCDE, Paris : OCDE.

PRUNK J. (1996) : Une brève histoire de la Slovénie, Ljubljana : Grad.

SVETLIK I., BARLE A. (2000) : «Curricular reforms: the case of Slovenia ». In GABRŠC`EK S. (ed.), DIMC N. (ed.).

\section{NOTES}

1. Le territoire slovène est à la croisée de l'Europe centrale, de la Méditerranée, de la Péninsule balkanique et de l'Italie. En tant que telle, la Slovénie est aujourd'hui membre de l'Union européenne, après avoir fait partie de la Yougoslavie, et «son histoire a toujours été celle d'une région de transit » (Prunk, 1996, 7). 
2. La structure fondamentale de l'éducation slovène est analogue à celle d'autres systèmes européens intégrés. NDLR : Voir le rapport Profil de la politique linguistique éducative Slovénie 2003, ministère de l'éducation, des sciences et du sport de Slovénie, p. 85 : www.coe.int/t/dg4/ Linguistic/Source/Country_Report_Slovenia_FR.pdf.

3. Commission européenne (1996). Dix années de réformes au niveau de l'enseignement obligatoire dans l'Union européenne, Bruxelles, p. 7.

4. Elle est la $66^{\mathrm{e}}$ en Europe selon le 2010 World University Ranking (http://www.4icu.org/topEurope) et la $41^{\mathrm{e}}$ en Europe selon le Webometrics Ranking of World Universities. Voir: http:// www.webometrics.info/top100_continent.asp?cont=Europe.

5. Voir : www.stat.si/novica_prikazi.aspx?id=1303.

6. Parmi les pays de l'OCDE, les élèves recevaient en 20057585 heures d'enseignement en moyenne entre 7 et 15 ans. Avec ses 6686 heures d'enseignement, la Slovénie est plutôt dans le bas du classement, avant la Finlande et la Norvège. Les élèves français, par exemple, sont dans le haut du classement avec 8437 heures d'enseignement (OCDE 2007, 369). La population slovène reçoit ainsi une année d'éducation de moins que la moyenne des populations des pays de l'OCDE et près de deux années d'enseignement de moins que la population du même âge en France.

\section{INDEX}

Mots-clés : réforme, système éducatif

Index géographique : Slovénie

\section{AUTEURS}

\section{SLAVKO GABER}

Professeur associé en sociologie de l'éducation à l'Université de Ljubljana, Slovénie. Ses domaines d'expertise sont les questions de qualité et d'équité, l'éducation comparée, la gouvernance, la gestion et l'assurance qualité en éducation. Expert en matière de réformes des politiques éducatives, il a conduit ou contribué à de nombreux projets en Serbie, au Monténégro, en Macédoine et en Bosnie-Herzégovine. Il est membre du réseau NESSE.

\section{VERONIKA TAŠNER}

Doctorante en sociologie et assistante en sociologie de l'éducation à l'Université de Ljubljana. Ses travaux portent sur la méritocratie, l'équité et les inégalités en éducation. 